\title{
Increasing of Equipment Efficiency by Intensification of Technological Processes
}

\author{
Anatolii Fesenko1, Yevheniia Basova1', Vitalii Ivanov², Maryna Ivanova', Fatyma Yevsiukova', \\ Magomediemin Gasanov ${ }^{1}$
}

\footnotetext{
1 Department of Technology of Mechanical Engineering and Metal-Cutting Machine Tools, Educational and Scientific Institute of Mechanical Engineering and Transport, National Technical University "Kharkiv Polytechnic Institute", 2 Kyrpychova str., 61002, Kharkov, Ukraine

${ }^{2}$ Department of Manufacturing Engineering, Machines and Tools, Faculty of Technical Systems and Energy Efficient Technologies, Sumy State University, 2 Rymskogo-Korsakova St., Sumy 40007, Ukraine

* Corresponding author, e-mail: e.v.basova.khpi@gmail.com
}

Received: 27 September 2018, Accepted: 16 October 2018, Published online: 04 December 2018

\begin{abstract}
Issues of technological processes' intensification and increase of technological equipment efficiency are of priority value in the modern engineering. Application of various methods of hydrodynamic drag on the working fluids in rotary-wheel machines allows increasing of processing efficiency to receive finely-dispersed homogeneous mixtures with necessary characteristics. In this article the issues, connected with the liquid flows' formation in the working zone of rotary-cavitating activator are studied and the main principles of energy impact while the usage of rotor with radial channels, supplied with the elements for receiving cavitating effects, are defined. Based on the offered methodology, experimental research was conducted and calculations of the main indexes, defining efficiency of energy impact on the working medium, were held. The substantiated estimation of efficiency of hydrodynamic machines work according to the dissipative power that allows to choose the proportion of hydrodynamic settings, which provide the highest intensity of cavitating processing of processed liquids, was presented. The practical value of the research is in the studying of ways of increasing of equipment efficiency through the possibility of technological process intensification.
\end{abstract}

\section{Keywords}

cavitator, intensification, hydrodynamic device, activator

\section{Introduction}

Technological processes' intensification and increase of technological equipment's efficiency in the engineering are the tasks of priority value, aimed at the search of optimal engineering solutions. For the present time, development of the high-efficient machines with high specific output and optimal energy and materials consumption became the basis for improvement of the products' quality, increase of productivity and decrease of energy consumption for conduction of various technological processes. Besides, demands of the high level of impact on the processed materials are settled to such machines.

\section{Literature review}

One of the most promising variants in contemporary engineering is the usage of various methods of activation of thinly-fluid products [1]. In a row of cases it allows to raise efficiency of processing or to create new, more progressive technological processes. At this, mechanical devices of dynamic type can be used. Activation takes place in them because of reaction of fluid for moving elements - rotating, vibrating or making more complicated movements [2-5]. For example, in rotor equipment, the energy which working fluids receive right in the device due to the centrifugal forces, appearing in the rotating rotor pockets, is used. Rotorcavitating activators (RCA) can be referred to such devices. The sense of their work is in creation of artificial cavitation in fluid areas for the usage of appearing cavitating effects for intensification of different physical and chemical processes in different fields of industry, for example, chemical, food processing, biochemical, engineering, oil-refining, etc.

Currently, in various industries the increasing application of hydrodynamic devices, due to which the conditions 
of cavitation in a fluid flow are created. For example, the new non-conventional machining method known as Bubble Machining is based on the process of cavitation [6]. The effect of ultrasound on generating and controlling the cavitation bubble of the grinding fluid during ultrasonic vibration honing was investigated [7]. In paper [8] a novel sharp interface approach for modeling the cavitation phenomena in incompressible viscous flows is described.

This ensures dispersion, homogenization, activation, acceleration of chemical reactions, heating of the working fluid $[9,10]$. These devices, depending on the technological purpose and embodied ideas of their authors, have different designs, but are designed for energy impact on the medium in the working area of plants.

The idea of using the cumulative effect of collapsing cavitation bubbles for the intensification of technological processes has led to creation of various structures of hydrodynamic cavitation devices.

Note that the technologies using cavitation are used for preparation of fuel mixtures, for making and recovering cutting fluids, for preparation of food mixtures and disinfection of water, for acceleration of chemical processes etc.

The hardware estimation procedure and optimization of parameters of working fluid cavitation processing are proposed in paper [11]. The basic aspects of preparation efficiency and using of water-fuel emulsion are reviewed and an example of estimation of energy parameters of hydrodynamic activator operation in obtaining water-fuel emulsion is supplied.

It is stressed that intensification of chemical-technological processes in rotor-cavitating activators is substantiated by reaching higher density values of hydrodynamic and hydroacoustic energy [9]. In the working volumes of device due to the short time intervals of influence the biggest part of power is localized in the flow of processed fluid. It takes place during the pressure and liquid flow speed pulsation, developed turbulence, intensive cavitation, pressure pulsation in local volumes of liquid, during pulsation and cavitating bubbles collapse, tough cumulative effect, high shearing and pulling forces [12].

At the same time, because of complexity and diversity of the whole complex of multi-factor effect on the processed liquid in rotor-cavitating activators all-sided researches of physical and chemical phenomena which take place during the processing of the liquid heterogenic mediums under effect of powerful pulsation flows, impulse acoustic cavitation, self-vibration and ripple effects are still actual.
Along with that rotor-cavitating activators have quite simple construction, their manufacturing does not require complicated technologies, they are trustworthy and effective in usage. Low energy capacity is substantiated by the fact that processing liquid is at the same time both the source and the object of hydromechanical vibrations. Mechanical energy is directly transferred into acoustic and cavitation energy and because of that device efficiency coefficient is quite high.

Rotor-cavitating activators proved themselves to be effective devices during intensification of hydromechanical and mass-exchanging processes in different spheres of industry [13]. They are more widely applied in engineering for manufacturing and recovery of fluids for cooling and lubrication (CLF).

The paper [14] presents a method for expanding the working range of separation elements, where the separation is conducted through the use of inertia particles. The presented dynamic separation elements work as the automatic control system.

The proposed experimental procedure has been used to investigate the liquid distribution inside the packed bed. Distilled water was adopted as a flowing medium and was injected into the column from a source point $[15,16]$.

An innovative method is proposed in the paper [17], which eliminates the low quality mesh elements and simultaneously introduces only slight distortion to the flow as well as contact heat transfer.

The authors of the paper [18] proposed the linear mathematical model and transfer functions, that were adapted to the drives of the technological equipment. It allows to perform the stability evaluation, quality of control and correction of the dynamic properties of the drive using the technical certificate data of the drive elements and devices.

Accordingly, among the most important tasks of contemporary industry one can distinguish the necessity of scientific substantiation and development of highly-effective technological devices, which provide effective influence on the working medium for intensification of complex influence on processing heterogeneous fluid.

The aim of this article is to substantiate the universal methodology for definition of working ability of rotor activators. Within the term "universal" the ability of application of this methodology during the analysis of similar constructions of cavitating equipment, intended to process of the thinly-fluid working medium, is meant. For the achievement of the settled goal it is necessary to conduct the analysis of settings of the processes, which are held in the second chamber (chamber under analysis). 


\section{Methodology}

For gaining of the settled aim it is necessary to analyze some issues, connected with formation of liquid flow in the working zone of rotor-cavitating activator and to define the main principles of energy impact while the usage of rotor with radial channels, supplied with elements for receiving of cavitating effects. So, the methodology for conduction of experimental researches and calculations of the main indexes which define effectiveness of energy impact on the working medium is expected to be worked out.

Design variables were defined according to the formulas, which consider energy expenditures in the working zone and results of preceding researches [11].

Power expenditures $(\mathrm{Np}$ ) for heating of the liquid, flowing through the activator is directly proportional to the dependence Eq. (1). This dependence allows to estimate indirectly intensification of the processes in the working zone according to the results of transformation of part of input energy into the dissipative one. Then, the dependence Eq. (1) allows to estimate the real power expenditures for heating of the liquid passing through the activator.

$N_{t}=K_{p} \cdot Q \cdot\left(T_{2}-T_{1}\right) \cdot T_{c} \cdot \rho$,

where $Q-$ is a flow rate through the dispersant (cum $/ \mathrm{h}$ ), $T_{1}$ and $T_{2}$ - fluid temperature at inlet and outlet of dispersant $\left({ }^{\circ} \mathrm{C}\right) ; T_{c}$ - heat capacity of working fluid $\left(\mathrm{kcal} / \mathrm{kg} \cdot{ }^{\circ} \mathrm{C}\right)$ - liquid density ( $\mathrm{kg} / \mathrm{cum}), K_{p}$ - is the coefficient of correspondence of dimensions (for water $K_{p}=1.164$ ), $\rho$ - liquid density (kg/cum); $K_{t}$ - dimensions coefficient.

$\left.K_{c}=Q \cdot(\Delta T),\left(\operatorname{cum} \cdot{ }^{\circ} \mathrm{C} / \mathrm{h}\right)\right)$.

Consequently, calculation of device heat load, under condition of working with various liquids can be done using Eq. (3)

$K_{w}=Q \cdot\left(T_{2}-T_{1}\right) \cdot T_{c} \cdot \rho$.

According to the device settings design power of the effect on the product $\left(N_{d}\right)$ is estimated according to Eq. (4)

$N_{d}=N_{c p}-\left(N_{f r}+N_{o f}+N_{i}\right)$.

At this, the free movement power $\left(N_{f r}\right)$, hydraulic power of the outlet flow $\left(N_{o f}\right)$ and expenditures for the internal losses $\left(N_{i l}\right)$ are subtracted from the power, consumed by the electromotor $\left(N_{c p}\right)$. Definite difficulties appear while definition of internal losses $\left(N_{i l}\right)$. It is also necessary take into account such constituents as losses for friction in the non-working pockets of chamber, which partially contribute to liquid heating. While various impact effects power can also be spent for chemical and physical transformations in the product [14]. Conduction of some extra researches is necessary for quantitative account of energy expenditures in these phenomena. In our research the information, received only from the experimental researches was used. It was enough for the practical realization of offered methodology [11].

Estimation of efficiency of the heat characteristics of cavitating processing of some definite liquid in the dispersant is recommended to be conducted according to two criteria: efficiency of heat emission $\left(K_{w}\right)$, that is characterized according to the level of cavitation effect on the working medium and heat density of the process $\left(E_{t}\right)[11]$ Eq. (5):

$$
E_{t}=\frac{N_{t}}{N_{q}} .
$$

In practice, during the hydrodynamic activators testing, it is quite easy to define such constituents as free-movement power $\left(N_{f r}\right)$ and hydraulic power $\left(N_{g}\right)$. So, accounting insufficient inaccuracies, the coefficient of energy transformation while heat emission can be presented as follows:

$$
E_{e f}=\frac{N_{t}}{N_{f r}+N_{q}} .
$$

During comparative analysis of hydrodynamic machines for liquid activation estimation of their efficiency (according to the level of energy transformation) is offered to be conducted through the evaluation of process according to the specific energy of dissipation. It means energy, spent for heat emission per unite of volume while passing of working product through the device

$W_{g}=\frac{N_{t}}{Q}(\mathrm{kWt} \mathrm{h/cum})$.

\section{Description of construction}

As the object of investigation rotor-cavitating activator "PKA-02-11", which has two working chambers, is analyzed in this article. The premixing of the product and its primary homogenizing takes place in the first chamber. The second chamber is used for intensive cavitating processing of the product in rotor channels. Due to this increase of device efficiency by means of technological process intensification is possible. In Fig. 1 construction of working zone, designed for intensive energy influence on working medium is shown. 


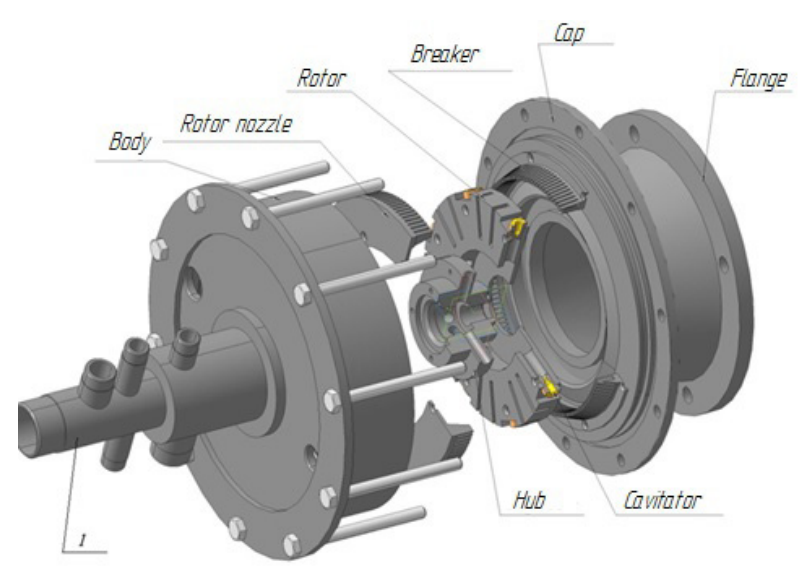

Fig. 1 The second chamber of activator "PKA-02-11"

Working liquid is supplied through the pipe 1, which is set on the rotor pin. The further acceleration of the liquid takes place in closed radial channels. At the end of the channel there is the nozzle. Cavitation appears in the flow, depending on design of the nozzle and at definite speed of liquid. At the end of the nozzle there is the breaker, which is constructed as the disk with inclined channels. It is designed for creation of turbulent motion of liquid, hydro blows and cavitation due to the sharp change of speed and direction of movement of the flow. To transmit extra energy to the liquid after passing the breaker, there is the nozzle on the rotor, which has radial slotted holes. Such design allows to create extra transformations of the flow into cavitation, blow and acoustic effects of the influence on the working medium (Fig. 2).

While the rotor movement liquid flow gets from cavitator to immovable breaker at the inlet. At this, the total velocity pressure is defined by the angle at which the flow meets the barrier and can be calculated according to Eq. (8), (Fig. 3).

$V_{s}=\operatorname{tg} \frac{V_{r}}{V_{c}}$.

The working scheme, adopted in terms of construction, is as follows: breaker has 150 riffles $(Z)$ 2-mm-width and located with the angle $60^{\circ}$. Such constructional decision was substantiated by the row of experiments, which proved the possibility to provide necessary frequency of vibrations of liquid flow velocity. Then, it is necessary to consider that with the frequency of rotation $n \mathrm{rpm}$ liquid flow from cavitator is interrupted $f$ times and its calculated value is defined according to Eq. (9)

$f=\frac{n \cdot Z}{60}=2950 \cdot \frac{150}{60}=7375(1 / \mathrm{sec})$.

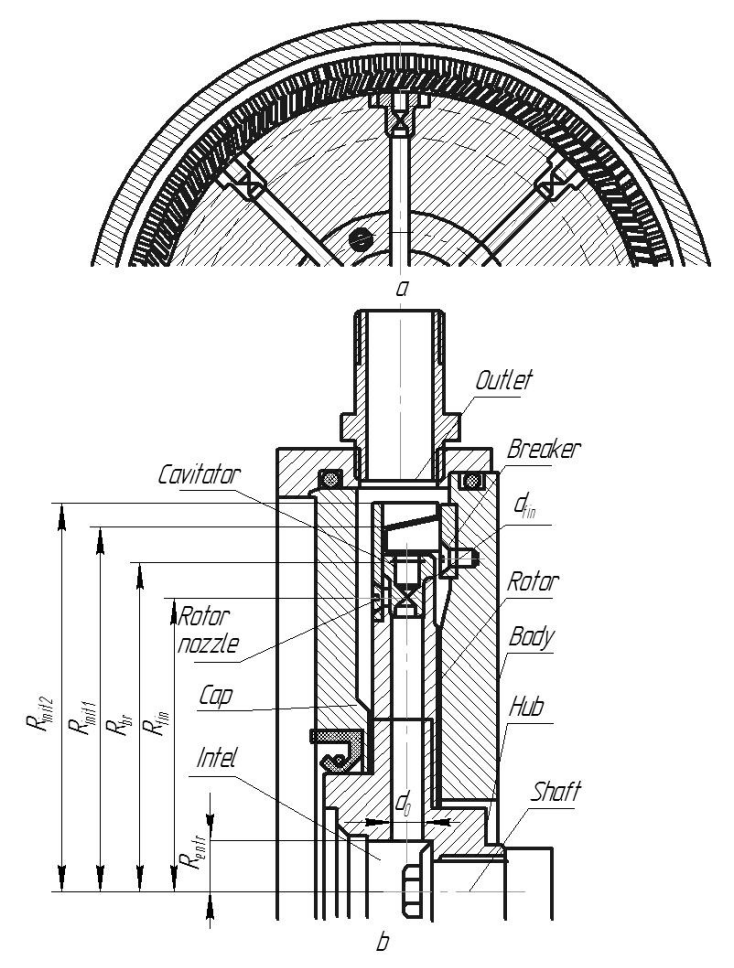

Fig. 2 Working zone of cavitating processing: a - cross-sectional view; b - longitudinal cross section

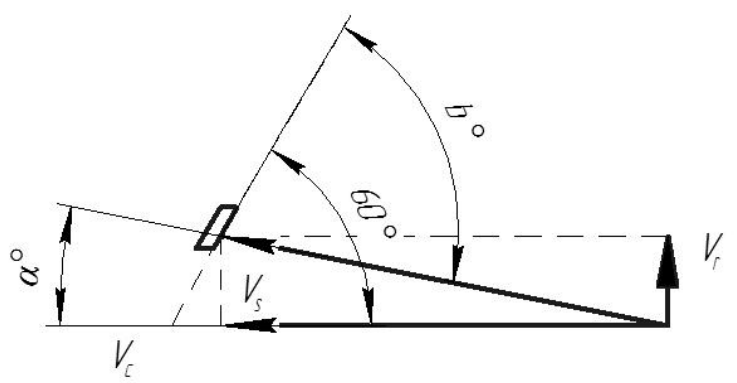

Fig. 3 Velocity distribution of the flow at the exit to the breaker: $\alpha$-slope angle of constituent of centrifugal force of the flow, $V r$ - radial constituent of liquid flow velocity; $V c$ - circumferential velocity of liquid flow; $\beta$ - angle, depending on $\alpha$

Pressure in the flow is changing, depending on relative position of cavitator and breaker. Qualitative characteristics of pressure changing $P$ on breaker which depends on time $t$ is shown in Fig. 4.

In this part the flow is partially losing its energy because of the resistance in the breaker channels. The size of this loss is defined by the coefficient of resistance in the sloped channels. When the flow gets into the channels of the rotor nozzle, the centrifugal effect starts working again and liquid gathers speed again (Fig. 4).

The size of this impulse $\left(F_{i \text { centr }}\right)$ of working liquid $\rho$ at constant rotation frequency $\omega$ is defined by differential 


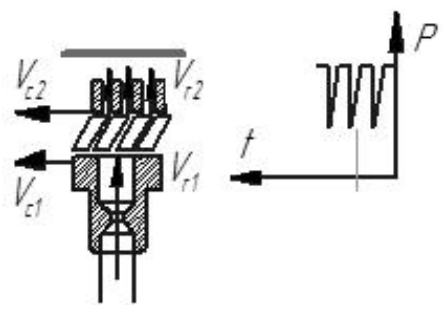

Fig. 4 Qualitative characteristics of pressure changing while passing of flow through the breaker

between the finite $\left(R_{\text {fin }}\right)$ and initial $\left(R_{\text {init }}\right)$ radiuses of the rotor and by the time of entering the channel $\tau$ Eq. (10):

$F_{i \text { centr }}=P_{i \text { centr }} \cdot \tau$,

where $P_{i \text { centr }}=\left(\rho \cdot \omega^{2} \cdot\left(R_{\text {fin }}-R_{\text {init }}\right)\right) / 2$.

In the rotor structure some extra channels for increasing of flow intensity at the exit to the breaker are provided (Fig. 5).

Channels are located at the left (back) rotor end face and have width $6 \mathrm{~mm}$ and depth 0-12 $\mathrm{mm}$. In total there are 26 channels, which are defined by the analytic optimization of studied construction.

Changes in the settings of the flow in the working zone of the chamber are shown in the structural scheme (Fig. 6). At this, energy, transmitted to the flow by centrifugal effect, in general is spent for overcoming of resistance in cavitator, breaker and at exit from working chamber.

While calculation of created pressure $P_{c}$ in the extra channels, it is necessary to consider, pressure of centrifugal effects $P$ and pressure in working chamber $P_{c h}$ Eq. (11)

$P_{c}=P+P_{c h}$.

Such rotor construction allows to bring additionally out to the breaker substantial volume of liquid, which had already been processed in cavitator. It intensifies the processes of hydrodynamic impact on working medium sufficiently. At this, frequency of vibrations and hydroblows on breaker at the outlet of liquid from each riffle is equal $f_{k}=7.4 \mathrm{kHz}$.

Kinetic energy Eq. (12), transmitted to the liquid while the rotor rotation, is spent for creation of pressure in the channel $E_{P}$, for radial movement of liquid in channel $E_{e f}$, for overcoming of resistance and cavitation in the nozzle, for overcoming of resistance and hydroblows in the breaker $E_{A}$, for overcoming of other types of resistance in the working zone $\Delta E$

$E_{o}=E_{P}+E_{e f}+E_{A}+\Delta E$.

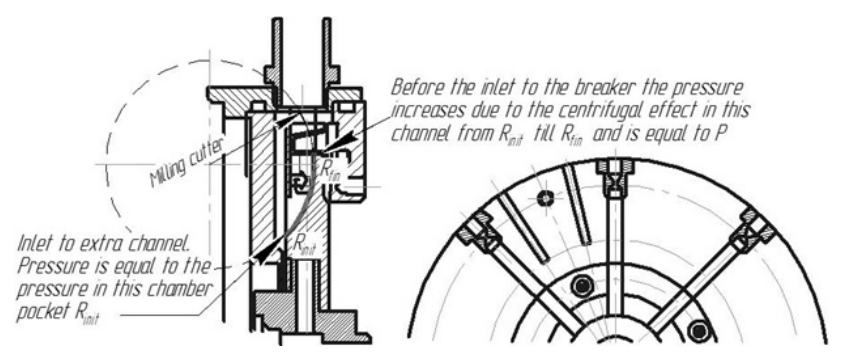

Fig. 5 Extra rotor channels and system of their location

Character of changes of flow setting in the working zone of the second chamber is shown in the structural scheme (Fig. 6).

Expenditures of liquid at the device outlet is defined by pressure losses in the working zone and resistance in the pipes (at the output). While increase of resistance at outlet expenditures reduce, and pressure in the working zone increases. Energy redistribution among impact effects takes place. Velocity in the channel decreases that leads to decrease of cavitation level in the nozzle. Quantity of liquid, taking part in the circular movement raises, stage of influence by whirling and pulsation at the breaker increases. When the outlet is closed movement of the liquid from the activator stops, pressure reaches its maximum value and dissipation of energy takes place, in general, on breaker and partially on rotor end faces.

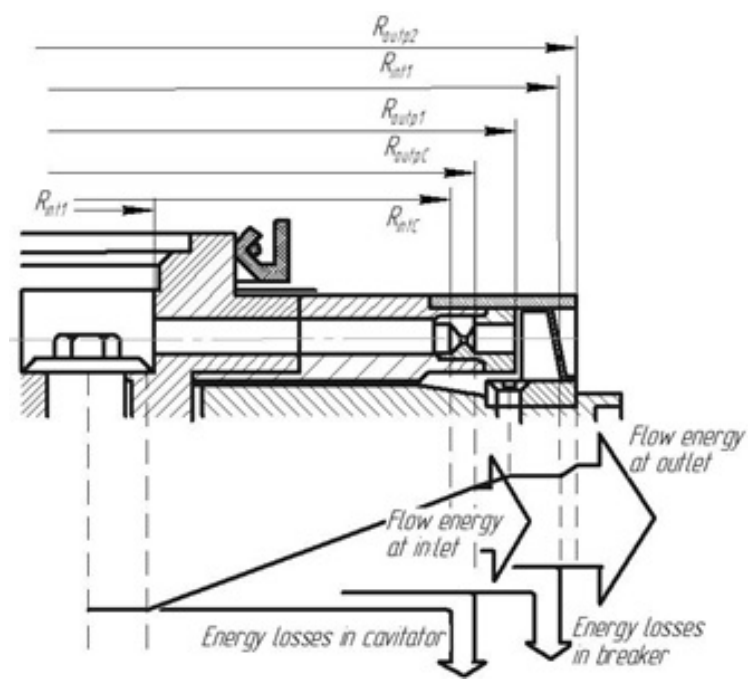

Fig. 6 Changes in flow settings in working chamber: $R_{\text {inpl }}$ - radius of input of the liquid flow in rotor; $R_{\text {inpC }}$ - radius of liquid flow input to cavitator; $R_{\text {outpC }}$ - radius of output of liquid flow from cavitator; $R_{\text {outpl }}-$ radius of liquid flow output from rotor; $R_{\text {inp2 }}$ - radius of input of the liquid flow into the nozzle; $R_{\text {outp } 1}$ - radius of liquid flow output from the nozzle 


\section{Description of construction}

For identification of hydraulic and energy indexes of the process in working zone experimental researches were held. Experimental device for studying of working zone of the second chamber of "PKA-02-11" is shown in Fig. 7.

For conduction of line of experiments activator was settled on the flange motor, with power $11 \mathrm{~kW}$. In such construction rotor is settled on the hub, connected with the motor shaft, due to which rotor is rotated with frequency $2950 \mathrm{rpm}$.

Processing of module liquid (water) during the closing of the valve at the inlet was done. For conduction of the hydrodynamic tests of experimental device we worked out automatic control system, which aloud to receive necessary data on the separate modules of the system, fixating the data in the PC. The outlet pressure was changing from 0.2 till $0.5 \mathrm{MPa}$ (pressure detectors were used). The pressure was controlled at the inlet and outlet (by Bourdon pressure gage of 0.4-class and with cock). Researches of productivity (by electronic water-meter), measuring differential of liquid temperature (by temperature detector) at inlet and outlet were held. Energy expenditures of investigating process were defined by A-meter, which was settled in the motor starter unit.

Results of the second chamber researches are presented in the diagrams (Fig. 8 and Fig. 9).

In the diagrams it is seen that increase of outlet pressure leads to decrease of productivity and increase of temperature differential at the inlet and outlet. At this, actual power remains almost unchangeable and dissipative power decreases.

Starting with the pressure $\mathrm{P} 2=0.4 \mathrm{MPa}$ specific energy of hydrodynamic impact increases.

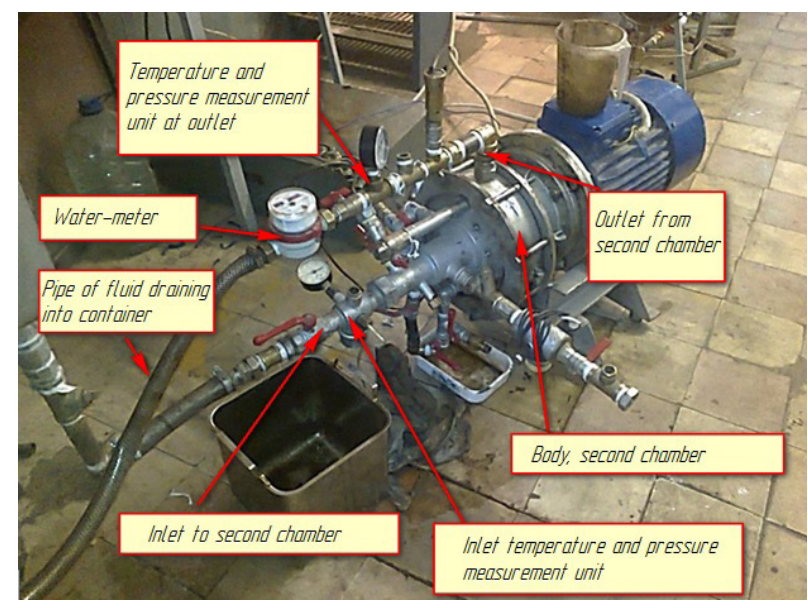

Fig. 7 Testing bench while measuring of the settings in the second chamber

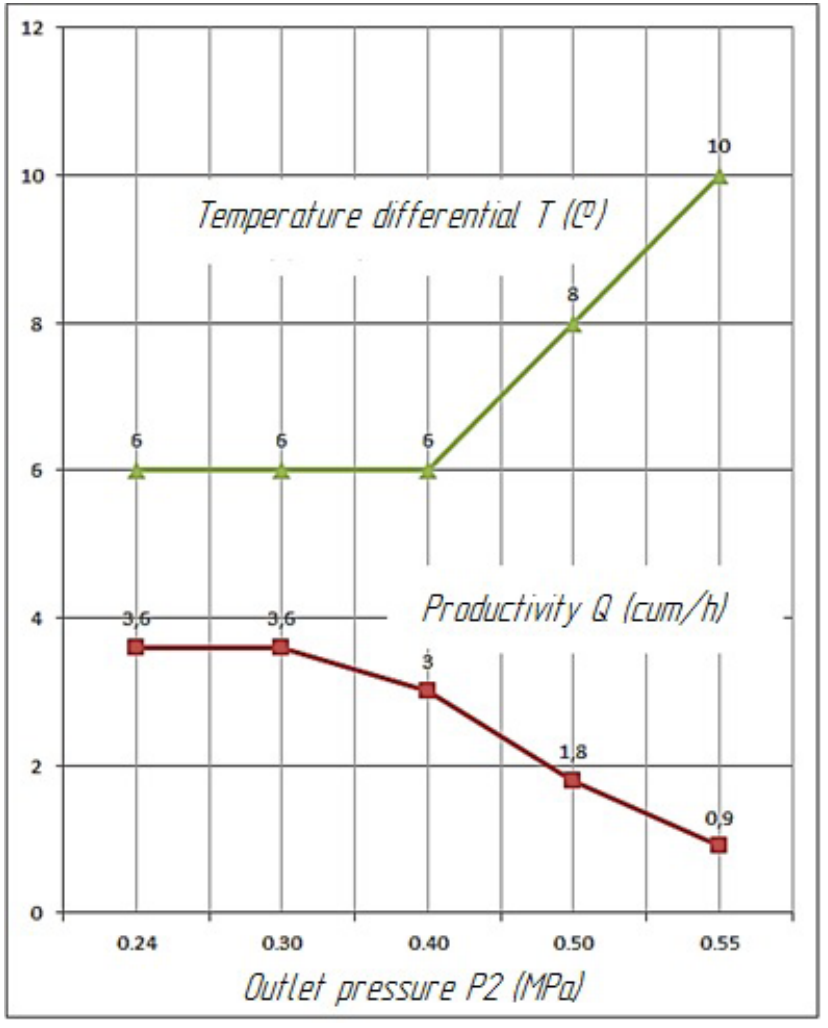

Fig. 8 Measuring settings

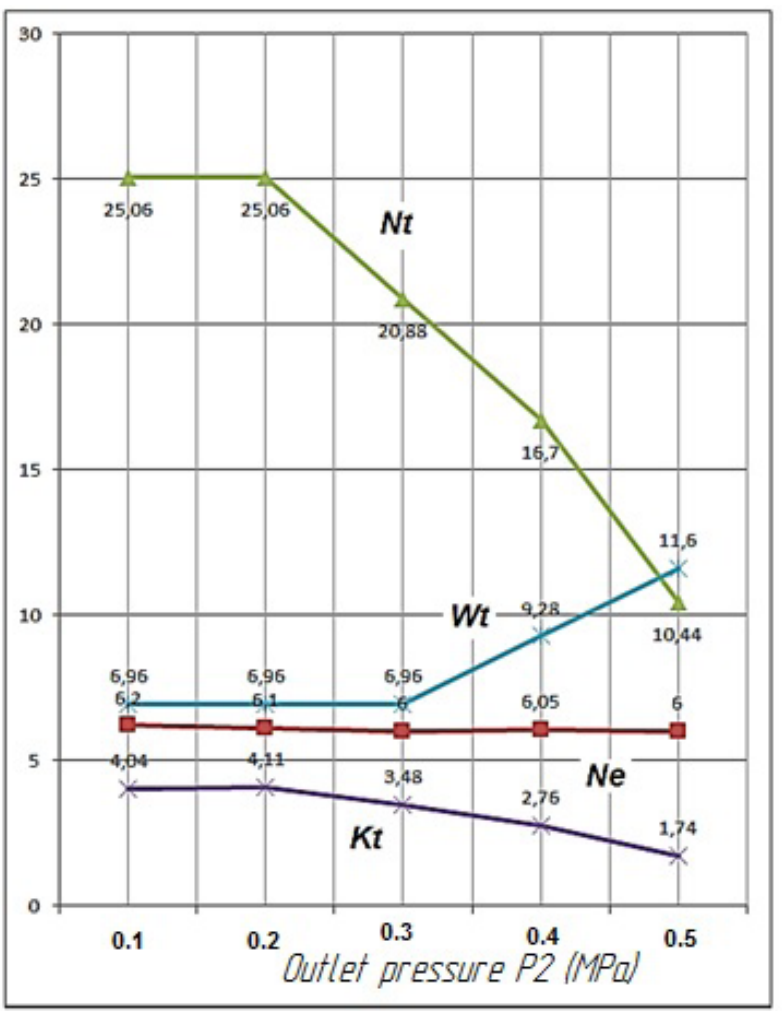

Fig. 9 Process indexes 


\section{Conclusion}

As the result of analysis and experimental testing of construction of working chamber of cavitating machine the main theoretical suppositions of energy distribution and hydrodynamic indexes of liquid activation while usage of rotor with radial channels and element for cavitating influence on working medium were proved.

\section{References}

[1] Oyewunmi, O. A., Kirmse, C. J. W., Pantaleo, A. M., Markides, C. N. "Performance of working-fluid mixtures in ORC-CHP systems for different heat-demand segments and heat-recovery temperature levels", Energy Conversion and Management, 148, pp. 1508-1524, 2017.

https://doi.org/10.1016/j.enconman.2017.05.078

[2] Becht, S., Franke, R., Geißelmann, A., Hahn, H. "An industrial view of process intensification", Chemical Engineering Processing: Process Intensification, 48(1), pp. 329-332, 2009.

https://doi.org/10.1016/j.cep.2008.04.012

[3] Kashid, M. N., Renken, A., Kiwi-Minsker, L. "Influence of Flow Regime on Mass Transfer in Different Types of Microchannels", Industrial \& Engineering Chemistry Research, 50(11), pp. 69066914, 2011

https://doi.org/10.1021/ie102200j

[4] Vardanega, R., Santos, D. T., Meireles, M. A. A. "Intensification of bioactive compounds extraction from medicinal plants using ultrasonic irradiation", Pharmacognosy Review, 8(16), pp. 88-95, 2014. https://doi.org/10.4103/0973-7847.134231

[5] Mohod, A. V., Gogate, P. R., Viel, G., Firmino, P., Giudici, R. "Intensification of biodiesel production using hydrodynamic cavitation based on high speed homogenizer", Chemical Engineering Journal, 316, pp. 751-757, 2017. https://doi.org/10.1016/j.cej.2017.02.011

[6] Vij, V., Goyal, K. "A Novel Approach to Hybrid AbrasiveCavitation Methods for Machining", European Journal of Advances in Engineering and Technology, 4(3), pp. 221-226, 2017. [online] Available at: http://www.ejaet.com/PDF/4-3/EJAET-4-3221-226.pdf [Accessed: 12 December 2017]

[7] Guo, C., Zhu, X. "Effect of ultrasound on dynamics characteristic of the cavitation bubble in grinding fluids during honing process", Ultrasonics, 84, pp. 13-24, 2018.

https://doi.org/10.1016/j.ultras.2017.09.016

[8] Michael, T., Yang, J., Stern, F. "A sharp interface approach for cavitation modeling using volume-of-fluid and ghost-fluid methods", Journal of Hydrodynamics, Ser. B, 29(6), pp. 917-925, 2017. https://doi.org/10.1016/S1001-6058(16)60806-5

[9] Fedotkin, I. M., Nemchin, A. F. "spol'zovanie kavitacii v tehnologicheskih processah" (The use of cavitation in technological processes), 1st ed., Vicha shkola, Kiev, Ukraine, 1984. (in Russian)

[10] Ivanov, V. M. "Toplivnye emul'sii" (Fuel emulsions), 1st ed., The USSR AS, Moscow, Russia, 1962. (in Russian)
Estimation of efficiency of hydrodynamic machines' work according to the dissipative power allows to choose the balance of the hydrodynamic settings (such as: reduced input pressure, output pressure, machine productivity, frequency and level of vibrations in the working zone), which provide the most intensive cavitating processing of process liquids.

[11] Fesenko, A., Yevsiukova, F., Basova, Y., Ivanova, M., Ivanov, V. "Prospects of Using Hydrodynamic Cavitation for Enhancement of Efficiency of Fluid Working Medium Preparation Technologies", Periodica Polytechnica Mechanical Engineering, 62(4), pp. 269-276, 2018. https://doi.org/10.3311/PPme.11877

[12] Chervjakov, V. M., Judaev, V. F. "Gidrodinamicheskie i kavitacionnye javlenija v rotornyh apparatah", (Hydrodynamic and cavitation phenomena in rotor machines) 1st ed., Mashinostroenie Publishers, Moscow, Russia, 2007. (in Russian)

[13] Promtov, M. A. "Pul'sacionnye apparaty rotornogo tipa: teoriya i praktika" (Pulsating apparatuses of rotary type: theory and practice), 1st ed., Mashinostroenie Publishers, Moscow, Russia, 2001. (in Russian)

[14] Pavlenko, I., Liaposhchenko, A., Ochowiak, M., Demyanenko, M. "Solving the Stationary Hydroaeroelasticity Problem for Dynamic Deflection Elements of Separation Devices", Vibrations in Physical Systems, 29, article number 2018026, 2018.

[15] Niegodajew, P., Wilczyński, M., Marek, M., Drobniak, S., Asendrych, D., Elsner, W., Gnatowska, R., Stempka, J. "A study of liquid spreading in laboratory scale random packing column with an optical method supplemented with liquid holdup characteristics", Experimental Thermal and Fluid Science, 96, pp. 162-168, 2018. https://doi.org/10.1016/j.expthermflusci.2018.03.008

[16] Ochowiak, M., Matuszak, M., Włodarczak, S., Ancukiewicz, M., Krupińska, A. "The modified swirl sedimentation tanks for water purification", Journal of Environmental Management, 189, pp. $22-28,2017$. https://doi.org/10.1016/j.jenvman.2016.12.023

[17] Sosnowski, M. "Computational domain discretization in numerical analysis of flow within granular materials", EPJ Web of Conferences: EFM17 - Experimental Fluid Mechanics 2017, 180, article number: 02095, 2018. https://doi.org/10.1051/epjconf/201817002095

[18] Sokolov, V., Krol, O. "Determination of Transfer Functions for Electrohydraulic Servo Drive of Technological Equipment", In: Ivanov, V., Rong, Y., Trojanowska, J., Venus, J., Liaposhchenko, O., Zajac, J., Pavlenko, I., Edl, M., Perakovic, D. (eds.) Advances in Design, Simulation and Manufacturing, DSMIE 2018, Lecture Notes in Mechanical Engineering, Springer, Cham, Switzerland, 2019, pp. 364-373.

https://doi.org/10.1007/978-3-319-93587-4_38 